\title{
El desafío de la traducción etnográfica en la Europa del siglo xxi: de la semiosis colonial a la decolonial ${ }^{1}$
}

\section{(TheChallengeofEthnographic Translationin Twenty-First Century Europe: From Colonial to Decolonial Semiosis)}

\author{
Christiane Stallaert ${ }^{2}$ \\ Universidad de Amberes, Bélgica \\ Universidad de Leuven, Bélgica
}

\section{resumen}

Si Europa siempre se ha caracterizado como un «espacio de traducción», esta característica se ha intensificado desde la segunda mitad del siglo xx por la llegada de inmigrantes y la globalización. En las metrópolis europeas de hoy el mayor desafío para la comunicación no es tanto la traducción interlingüística sino entre culturas. Proponemos el enfoque de «etnografía multisituada» como modo de acercamiento al estudio de la Europa superdiversa del siglo xx. La perspectiva multisituada nos permite detectar igualmente la transición desde una semiosis colonial a otra decolonial.

\section{abstract}

If Europe has always been known as a "space of translation," this feature has been intensified since the second half of the twentieth century by the arrival of immigrants and by globalization. In today's European cities the challenge of communication is not so much linguistic as it is cultural. I propose

1 Elaborado con base en la ponencia presentada por la autora en el IV Congreso Internacional de Lingüística Aplicada, llevado a cabo en mayo de 2013, en el Campus Omar Dengo, de la Universidad Nacional de Costa Rica. Recibido: 19 de abril de 2012; aceptado: 3 de julio de 2012.

2 Correo electrónico: christiane.stallaert@uantwerpen.be 
"multisited ethnography" as a way of studying superdiverse 21 st century Europe. A multisited perspective also enables us to detect the transition from a colonial semiosis to a decolonial semiosis.

Palabras clave: traducción cultural, Europa, globalización, decolonialidad Keywords: cultural translation, Europe, globalization, decoloniality

A finales de marzo de 2013, en Gante (Flandes, Bélgica) un jurado popular tuvo que pronunciarse sobre un caso de homicidio múltiple cometido por un joven de veinte años, que, cuatro años antes, sin aparente motivo había asesinado brutalmente a dos bebés y a una niñera, además de dejar una docena de heridos en una guardería infantil. Durante el juicio, el estado psíquico del asesino estuvo en el centro del debate. La falta de consenso entre los médicos forenses acerca del diagnóstico psiquiátrico se vio agravada por la imprecisión del concepto jurídico de «ontoerekeningsvatbaar» («incapacidad de culpabilidad» o «inimputabilidad»), que, según el derecho penal belga significa que el reo «no es responsable de sus actos debido a una enfermedad o deficiencia mental». Se intentó buscar una respuesta mirando hacia el país vecino, Holanda, país con el que Flandes comparte la misma lengua aunque no el ordenamiento jurídico. Sin embargo, en vez de solucionarse, el asunto se complicó, ya que a diferencia del ordenamiento jurídico belga que sólo prevé una respuesta en blanco y negro (sí o no), el derecho holandés define zonas «grises» y reconoce hasta cinco grados de inimputabilidad por enfermedad mental. Finalmente, ante la indecisión de los expertos, le tocó a un tribunal popular zanjar la cuestión y juzgar si el autor del crimen era imputable o no.

No había motivos para que este caso fuera tan complejo, ya que el juicio se desarrollaba estrictamente a nivel intralingüístico e intracultural, entre actores que compartían la misma lengua y cultura. El juicio se efectuó en Flandes, con un tribunal flamenco compuesto de jueces flamencos, las víctimas y los testigos eran todos flamencos, los expertos forenses eran flamencos así como el joven autor de los hechos. 
Aunque menos que en Flandes, el caso del asesinato múltiple también atrajo los medios francófonos del país. El tenor del debate era, sin embargo, diferente. Por un lado, en francés «irresponsable de ses actes» —el equivalente jurídico de «ontoerekeningsvatbaar»- no se asocia espontáneamente con un problema de salud mental mientras que, por otro lado, el término neerlandés no evoca de forma directa la idea de exención de responsabilidad. Dentro de un mismo ordenamiento jurídico definido en dos lenguas nacionales —el neerlandés y el francés - un mismo concepto da lugar a connotaciones distintas.

Obtenemos un cuadro aún más diferente si nos desplazamos a Bruselas, situada a cincuenta kilómetros de Gante, lugar del citado juicio. Zona de contacto entre las dos grandes comunidades lingüístico-culturales de Bélgica, la flamenca (de habla neerlandesa) y la valona/francófona (de habla francesa), Bruselas ha evolucionado en los últimos decenios de una ciudad oficialmente bilingüe aunque mayoritariamente francófona hacia una ciudad caracterizada por la «superdiversidad $»^{3}$ donde el multilingüismo se ha convertido en norma. Mientras que el inglés ha sobrepasado al neerlandés en número de hablantes, existe un $10 \%$ de habitantes de Bruselas que no habla ninguna de las tres lenguas mayoritarias (francés, inglés o neerlandés). Asimismo se ha registrado un avance espectacular del árabe, lengua hablada por casi un $20 \%$ de la población (el $10 \%$ en 2000) $)^{4}$.

Si tenemos en cuenta este último dato — el avance espectacular del árabe como primera, y en algunos casos, única lengua de los habitantes de Bruselas a inicios del siglo xxi - nos encontramos ante un nuevo desafío de traducción de conceptos. El siguiente caso ocurrido en Francia nos puede servir de ejemplo. En 2010 se descubrió que un argelino, que en 1999 había adquirido la nacionalidad francesa, tenía cuatro mujeres y que cada una recibía un subsidio social del Estado

3 Steven Vertovec, «Super-Diversity and Its Implications», Ethnic and Racial Studies 29, 6 (2007): 1024-54.

4 BRIO-taalbarometer Brussel, 20 de marzo de 2013, <http://www.briobrussel.be/ned/resourcedetail.asp?ResourceId=632>. 
como madre soltera. Cuando el ministro de Interior francés amenazó con quitarle la nacionalidad francesa por delito de «poligamia», el imputado se defendió alegando que tenía cuatro «amantes», práctica no tipificada como delito bajo la ley francesa ${ }^{5}$. Recientemente, en abril de 2013, Francia aprobó en medio de protestas masivas la ley de matrimonio homosexual, conocida como «ley del matrimonio para todos» («Loi du mariage pour tous»). Aunque las protestas procedían de sectores católicos conservadores, algunos periodistas se preguntaban si en nombre de la «igualdad» republicana habría que incluir también en el futuro la opción musulmana del matrimonio poligámico ${ }^{6}$. El debate en Francia coincidía con las protestas en apoyo de Amina, una joven tunecina que se había manifestado en Facebook contra la moral estricta impuesta en su país por los islamistas ${ }^{7}$. El haberse retratado en torso desnudo le podía costar la muerte por lapidación. De forma preventiva, la familia hizo «internar» a la joven ${ }^{8}$. ¿Será que, de acuerdo con las normas islamistas (¿islámicas?), Amina «no era responsable de sus actos por enfermedad mental»?

De la misma índole es el debate hoy día en Europa en torno al concepto de «acoso sexual». Mientras que Bélgica tipificó este delito en 2002 (ley de 11 de junio de 2002), Francia, donde el «harcèlement (sexuel)» había sido introducido en el código penal en 1992, decidió abrogar la ley en mayo de 2012 por falta de precisión en la definición de «los elementos constitutivos del delito» ${ }^{9}$. La reforma en Francia coincidía con una visita oficial del gobierno belga en Marruecos, en abril de 2012, en cuya ocasión el primer ministro marroquí se

5 «lk ben niet polygaam, ik heb maîtresse'», De Standaard, 26 de abril de 2010, <http://www.standaard.be/artikel/detail.aspx?artikelid=LF2PFGF2>.

6 Mia Doornaert, «Waarom Frankrijk zo massaal tegen het homohuwelijk protesteert», De Standaard, 4 de abril de 2013, p. 38-39, <http://www.standaard.be/artikel/detail.aspx?artikelid=D MF20130403_00528602>.

7 <http://femen.org/en/news/id/294>.

8 De Standaard, 26 de marzo de 2013, p. 18.

9 Le Conseil constitutionnel censure la loi sur le harcèlement sexuel, Le Monde, 4 de mayo de 2012, $<$ http://www.lemonde.fr/societe/article/2012/05/04/le-conseil-constitutionnel-censure-la-loi-surle-harcelement-sexuel_1695710_3224.html>. 
negó a estrecharle la mano a la ministra de Justicia belga y durante la visita no le dirigió ni la mirada ni la palabra. ¿Sería por miedo de ser acusado de acoso sexual? Es lo que irónicamente se preguntaban algunos periodistas belgas. La cuestión, sin embargo, no es baladí si tenemos en cuenta el alto porcentaje de población de origen marroquí o musulmana en Bruselas. Si Francia tuvo que abrogar la ley sobre el acoso sexual por falta de precisión terminológica, qué pensar entonces de un escenario donde el consenso terminológico se ha de establecer entre tradiciones culturales y religiosas muy diferentes, reunidas todas bajo una misma ley. En un entorno multicultural, los conceptos se convierten en resbaladizos y de contornos inciertos. ¿Qué significa hoy en Bruselas —o en cualquier metrópoli europea caracterizada por la superdiversidad a consecuencia de los flujos migratorios recientes - el concepto de «emancipación» o de «religión»? ¿Cómo entender el derecho a la libertad religiosa? ¿Incluye el de institucionalizar la posición subalterna de la mujer? Y el de la libertad de expresión, ¿incluye de criticar opciones religiosas contrarias a dicha libertad? Estas cuestiones de difícil solución, síntoma de la incertidumbre que provoca la experiencia multicultural, ocupan el debate público en la Europa del siglo xxi.

Desde un punto de vista occidental, solemos ver «la globalización» como un fenómeno nuevo que, para estudiarlo, requiere una metodología nueva. Los antropólogos, conscientes del nuevo desafío que significa el mundo «globalizado», desarrollaron nuevos conceptos y enfoques metodológicos. Dentro de este contexto, George E. Marcus recomienda abandonar la práctica etnográfica clásica del trabajo de campo enraizado en un solo lugar, proponiendo un nuevo modelo de investigación más adecuado a un mundo de flujos y paisajes compuestos por realidades discontinuas. En su modelo de «etnografía multisituada», Marcus parte de la idea de que las lógicas culturales que el antropólogo espera descubrir son producidas en múltiples lugares y la estrategia para estudiarlas consistiría en «seguir literalmente las 
conexiones, asociaciones y supuestas relaciones $»^{10}$. Esta técnica del trabajo de campo multisituado se inscribe en el análisis de sistemamundo descrito por Wallerstein implicando, según Marcus ${ }^{11}$, un enfoque interdisciplinario y la definición de nuevos objetos de estudio. Lo interesante para el tema que nos ocupa es que el modelo de Marcus otorga un papel fundamental a la traducción. Comparad con la etnografía tradicional, en la etnografía multisituada,

[t]he function of translation (from one cultural idiom or language to another) is enhanced since it is no longer practiced in the primary, dualistic «them-us» frame of conventional ethnography but requires considerably more nuancing and shading as the practice of translation connects the several sites that the research explores along unexpected and even dissonant fractures of social location. Indeed, the persuasiveness of the broader field that any such ethnography maps and constructs is in its capacity to make connections through translations and tracings among distinctive discourses from site to site ${ }^{12}$.

Según la metodología propuesta por Marcus, estudiamos la trayectoria a lo largo del tiempo y del espacio del concepto ibérico de «casticismo» ${ }^{13}$. Inicialmente, en el contexto ibérico medieval, «casta» era sinónimo de «especie» o «linaje» y servía como concepto genérico para denominar las categorías etnorreligiosas que convivían en la

10 George E. Marcus, Ethnography Through Thick and Thin (Princeton: Princeton University Press, 1998), 81.

11 Marcus, 86.

12 Marcus, 84.

13 Otro ejemplo de la metodología de Marcus lo ofrece el libro coordinado por Carol Gluck y Anna Lowenhaupt Tsing, Words in Motion: Toward a Global Lexicon (2009). En esta colección de ensayos, cada autor sigue un concepto en su periplo a lo largo del tiempo y del espacio. Entre los conceptos estudiados se encuentran seguridad en Brasil, responsabilidad en Japón, secularismo en Marruecos o hijab en Francia. Ver Christiane Stallaert, «Castas, razas, etnias. Circulación intercontinental de conceptos y personas», en Kristien Vanden Berghe (ed.), El retorno de los galeones. Literatura, arte, cultura popular, historia (Frankfurt/Main: Peter Lang, 2011) 77-94. 
Península Ibérica ${ }^{14}$. Gradualmente, la noción de «casta» adquirió la connotación de «pureza», en el sentido genealógico o racial, es decir, de linaje no contaminado por sangre ajena. Con el proyecto imperialista, el ideal casticista se exportó a otros continentes, principalmente a América, donde el concepto de «casta» se emancipó de su contexto ibérico para adoptar nuevos significados más acordes al nuevo entorno. Mientras que en la Península Ibérica «casta» (y la calidad de «castizo») se había convertido en prerrogativa de los cristianos étnicamente puros (cristianos viejos), en el continente americano al compás del imparable mestizaje de blancos, negros e indios, el concepto de casta dejó de referirse a la pureza étnica para significar preferentemente la mezcla biológica (en plural, «las castas»). Si al principio designaba categorías sociales estancas y herméticas, pasó a significar el proceso dinámico e imparable de las hibridaciones. En su migración hacia el Nuevo Mundo cruzando el Atlántico, el concepto de «casta» se transculturó por influencia de la nueva realidad humana emergida de la sociedad colonial muy alejada de la realidad peninsular.

George Marcus diseñó su metodología de la etnografía multisituada para el estudio de los nuevos «paisajes» antropológicos surgidos a raíz de la globalización. Su uso del término de «paisaje» (landscape) remite a la obra del antropólogo Arjun Appadurai ${ }^{15}$ en cuya terminología el sufijo «-scape» («ethnoscape», «ideoscape», etc.) da cuenta de realidades diaspóricas en un mundo globalizado donde las trayectorias seguidas por personas, ideas o productos se vuelven cada vez menos isomorfas. El movimiento diaspórico disyuntivo hace que los conceptos que conforman un «ideoscape» se vayan incrustando en nuevos contextos muy diversos creando caleidoscopios terminológicos. Appadurai distingue entre la «genealogía» de los conceptos, definida como su circulación geográfica, y la

14 Christiane Stallaert, Etnogénesis y etnicidad en España. Una aproximación histórico-antropológica al casticismo (Barcelona, Anthropos, 1998); y Ni una gota de sangre impura. La España inquisitorial y la Alemania nazi cara a cara (Barcelona, Galaxia Gutenberg/Círculo de Lectores, 2006).

15 Arjun Appadurai, Modernity at Large. Cultural Dimensions of Globalization, primera edición: 1996 (Minneapolis: University of Minnesota Press, 2008). 
«historia» de los mismos, definida como el proceso de su domesticación en una práctica local ${ }^{16}$. Con respecto al «casticismo», establecer el vínculo entre historia y genealogía del concepto nos transporta, primero, del marco ibérico al colonial y, segundo, del colonial a otro de-colonial, este último definido desde el punto de vista de la antigua colonia como «un diálogo con el pasado colonial y no simplemente el desmantelamiento de los hábitos o modos de vida coloniales» ${ }^{17}$. Reconstruir etnográficamente el «ideoscape»-el paisaje diaspórico conceptual- del casticismo nos obliga a transitar a través de tiempo y espacio pasando no por uno sino por dos momentos de globalización. En cada una de estas globalizaciones el «ideoscape» del casticismo se enriquece con nuevos significados importados desde los contextos históricos y geográficos cambiantes.

Hemos aludido a la migración del concepto de «casta» durante la primera globalización a partir de 1492. Para España, la «segunda globalización» (comúnmente designada como «la»globalización) irrumpe con fuerza en la década de 1990, coincidiendo con medidas nacionales de liberalización económica que trajeron consigo la privatización e internacionalización de empresas estatales y el boom en el sector de la construcción, que propició la reactivación del sistema migratorio transatlántico, en dirección contraria esta vez al colonial. Los inmigrantes iberoamericanos llegaron a situarse a la cabeza de la población extranjera en España ascendiendo en la Comunidad de Madrid al $46 \%$ del total de extranjeros (cifras de $2008^{18}$ ).

Si el «casticismo» ha sido el concepto clave en la construcción de la identidad española ${ }^{19}$, es interesante examinar cómo el «ideoscape» —el paisaje conceptual — del casticismo se ha visto afectado por el nuevo contexto humano de la «neohispanoamericanización»

\footnotetext{
$16 \quad$ Appadurai, 17.

17 Appadurai, 89.

18 Christiane Stallaert, «Castas, razas, etnias. Circulación intercontinental de conceptos y personas», en Kristien Vanden Berghe (ed.), El retorno de los galeones. Literatura, arte, cultura popular, historia (Frankfurt/Main: Peter Lang, 2011) 77-94.

19 Stallaert (1998).
} 
en suelo peninsular. Hemos estudiado este tema a partir de un trabajo de campo en Madrid y Toledo ${ }^{20}$. Me limito a destacar que la etnografía multisituada reveló la pugna o tensión entre dos modelos de sociedad; por un lado, la sociedad «celular» propia de la era globalizada caracterizada por redes y flujos transfronterizos disyuntivos y, por otro, la sociedad «vertebrada», vestigio del ideal de EstadoNación. La terminología para caracterizar esta oposición procede de Appadurai, quien observa que «[m]odern nation-states recognize their common belonging to the vertebrate world and, like the last dinosaurs, see that they are in a desperate struggle for survival as a global formation» ${ }^{21}$. Según nuestro análisis, el casticismo, espina dorsal de la «España vertebrada», aparece como el dinosaurio que lucha desesperadamente por la supervivencia bajo la presión de la nueva globalización. Según Appadurai, lo propio de la sociedad «vertebrada» del Estado Nación es que este sistema se apoya en un sistema semiótico de comunicación compuesto por un conjunto limitado de normas y signos mutuamente reconocidos por los miembros ${ }^{22}$.

Esta observación retrotrae al principio del presente texto contextualizado en la creciente diversidad de lenguas y culturas con la que se ven confrontados los países europeos de hoy. En la mayoría de estos países, por efecto de la globalización, se está perturbando el sistema semiótico compartido que garantizaba la relativa estabilidad del sistema de sociedad «vertebrada» conocida como «Estado Nación». Visto así, Europa se encuentra en un momento de transición, donde los paisajes conceptuales (ideoscape) tradicionales están bajo tensión. Por analogía con el ejemplo del «casticismo», que por efecto del colonialismo (o primera globalización) fue exportado a

20) Stallaert (2011).

21 Stallaert (2006) 21.

22 «The system of nation-states has relied from the start on a system of semiotic recognition and communication, composed of such simple items as flags, stamps, and airlines and by much more complex systems such as those of consulates, ambassadors, and other mutual forms of recognition. Such vertebrate systems, of which the system of nation-states may be the largest and most extensive in scale, are not necessarily centralized or hierarchical. But they are fundamentally premised on a finite set of coordinated, regulative norms and signals» (Appadurai (2006), 25). 
América donde se transculturó para luego regresar a España a finales del siglo xx, hoy Europa se ve confrontada con el «retorno» de sus propios conceptos e ideales. Junto con los inmigrantes africanos, latinoamericanos o asiáticos los conceptos históricos que acompañaron la génesis del Estado-Nación europeo regresan transculturados a Europa, resemantizados a lo largo del periplo migratorio. El gran desafío para la Europa «globalizada» de hoy es cómo garantizar un marco semiótico suficientemente estable y mutuamente reconocible y reconocido en medio de un paisaje humano cada vez más diverso e inestable. En otras palabras y aludiendo a los ejemplos antes citados, ¿cómo insertar, por ejemplo, una concepción «musulmana» de «emancipación», «libertad», «igualdad» en el ideoscape doméstico europeo desde donde estos mismos conceptos fueron exportados hacia el mundo como parte de un proyecto político determinado?

El precedente de la primera globalización sirve de referente histórico para comprender la disyuntiva ante la cual se halla la Europa del siglo xxi. La llegada de los europeos (españoles y portugueses) al continente americano a finales del siglo xv significó la importación de un sistema de signos en un entorno nuevo, creando una «semiosis colonial $»^{23}$, fruto de la dialéctica entre signos procedentes de diferentes tradiciones culturales. Desde las primeras fechas de la llegada de los europeos al llamado Nuevo Mundo existen testimonios sobre el conflicto semiótico generado. La «semiosis colonial» queda patente en la Carta de Pedro (o Pêro) Vaz de Caminha, de 1500 , donde el autor escribe que las mujeres indígenas andaban con «sus vergüenzas tan desnudas y con tanta inocencia descubiertas, que en eso no había vergüenza alguna ${ }^{24}$. Una lectura sintomá-

23 Walter Mignolo, «Colonial and Postcolonial Discourse: Cultural critique or Academic colonialism?», Latin American Research Review XXVIII, 3 (1993): 120-134.

24 En portugués: «Andam nus, sem cobertura alguma. Nem fazem mais caso de encobrir ou deixa de encobrir suas vergonhas do que de mostrar a cara. Acerca disso são de grande inocência». También: «Ali andavam entre eles três ou quatro moças, bem novinhas e gentis, com cabelos muito pretos e compridos pelas costas; e suas vergonhas, tão altas e tão cerradinhas e tão limpas das cabeleiras que, de as nós muito bem olharmos, não se envergonhavam»; véase: <http://www.ufrgs. br/proin/versao_1/caminha/index02.html>. 
tica de testimonios tempranos de la primera globalización revela el choque semiótico entre la cultura europeo-cristiana, inscrita en la lengua del colonizador, y la realidad indígena que se intenta describir. Según Mignolo $^{25}$, una característica de la semiosis colonial es su dimensión híbrida, resultante del traslado del signo (originado en un contexto geográfico y cultural determinado) a un contexto diferente. En el proceso de migración, el signo original procedente del centro de la modernidad europea con su ideal de lo puro y homogéneo se convierte en copia contaminada, impura, imperfecta o mestiza en la situación colonial. Recordemos que lo impuro, lo «sucio» ha sido definido por Mary Douglas como «lo que está fuera de lugar», fuera de contexto, característica de lo foráneo y del forastero ${ }^{26}$.

Desde una perspectiva histórica, este choque semiótico fue el motor de la transculturación de conceptos europeos introducidos en una realidad distinta, en un «Mundo Nuevo». Aparte del conceptode «casta», otro ejemplo es el de «criollo», palabra de etimología incierta aunque asociada inicialmente con españoles y esclavos negros nacidos en América, es decir, fuera del territorio tribal o étnico. Aunque originalmente sin ninguna connotación de mestizaje o mezcla racial/étnica, hoy en día «creolización» evoca la idea de mestizaje, de producto del contacto e intercambio intenso entre culturas de distintas procedencias.

En su Historia natural y moral de las Indias (1590), el jesuita José de Acosta estaba consciente de que el viaje de los conceptos europeos hacia el Nuevo Mundo ponía en movimiento sus significados hasta hacer desaparecer la relativa estabilidad denotativa que parecían poseer en el contexto del Viejo Mundo. El conocimiento empírico del Nuevo Mundo y el contacto con su enorme diversidad contradice ciertas «verdades» europeas, lo cual obliga a revisar ciertas leyes concebidas desde Europa introduciendo una perspectiva

25 Walter Mignolo, The Movable Center: Geographical Discourses and Territoriality During the Expansion of the Spanish Empire', en Ana del Sarto, Alicia Ríos y Abril Trigo (eds.), The Latin American Cultural Studies Reader (Durham, NC: Duke University Press, 2004) 262-290 (289).

26 Mary Douglas, Purity and Danger (Londres: Routledge, 1966) 52. 
múltiple y transcontinental ${ }^{27}$. Esta conciencia, presente en José de Acosta y otros cronistas del Nuevo Mundo, rompe con la euro-normatividad (el tomar Europa como única norma del sistema semiótico). En su descripción del Nuevo Mundo, Acosta se muestra capaz de adoptar una perspectiva des-centrada, no eurocéntrica. Hablando de los vientos en la región equinoccial (Libro segundo, capítulo 13), escribe desde una perspectiva cambiante, tomando una vez Europa y otra vez América como referencia normativa: «Lo cual en Europa es al revés», y unos párrafos más bajo: «Mas en el Pirú y en toda la Equinocial [sic] es al contrario» ${ }^{28}$.

Recordemos que el concepto de «semiosis colonial» remite a un proceso de producción de significados relacional e interaccionista ${ }^{29}$.

Colonial semiosis is the general term to indicate a network of semiotic processes in which signs from different cultural systems interact in the productions and interpretation of hybrid cultural artifacts. In colonial semiosis the meaning of a sign no longer depends on its original cultural context (for instance, Castilian, or Amerindian, or Chinese), but on the new set of relations generated by communicative interactions across cultural boundaries.

Esta producción de nuevos significados en el contexto de la colonización de Latinoamérica es el resultado de un proceso de

27 Así, uguiándonos no tanto por la doctrina de los antiguos filósofos cuanto por la verdadera razón y cierta experiencia» (Acosta 2008:45), la experiencia en el Nuevo Mundo enseña que «la Tórrida Zona es humidísima, y que en esto se engañaron mucho los antiguos», siendo esta verdad además comprobada en la India Oriental según el testimonio obtenido en las cartas de otros jesuitas: «Lo mismo está observado en la India Oriental, y por la relación de las cartas de allá parece ser así» (Acosta 2008:46 y 47); «Siendo así que en las causas naturales y físicas no se ha de pedir regla infalible y matemática sino que lo ordinario y muy común eso es lo que hace regla, conviene entender que en ese propio estilo se ha de tomar lo que vamos diciendo: que en la Tórrida hay más humidad que en esotras regiones, y que en ella llueve cuando el sol anda más cercano, pues esto es así según lo más común y ordinario. Y no por eso negamos las excepciones que la naturaleza quiso dar a la regla dicha, haciendo algunas partes de la Tórrida sumamente secas, como de la Etiopía y de gran parte del Pirú lo hemos visto» (Josef de Acosta, Historia Natural y Moral de las Indias, edición crítica de Fermín del Pino-Díaz (Madrid: CSIC, 2008) 51).

28 Josef de Acosta, 56-57.

29 Mignolo (2004) 262. 
traducción, entendida como «el proceso de relacionar o crear convergencias, homologías o equivalencias entre elementos inicialmente dispares ${ }^{30}$; la traducción vista como un proceso de inter-relacionamiento de lenguajes semióticos distantes, y puede ser estudiada etnográficamente de acuerdo con una metodología multisituada.

Desde el observatorio eurocéntrico ha resultado difícil darse cuenta de los procesos de transculturación semiótica que se operaron en las culturas y lenguas de los nuevos mundos colonizados. Es ahora, cuando por efecto de la segunda globalización los conceptos occidentales ya transculturados vuelven hacia Europa, que el viejo centro del sistemamundo se ve confrontado con los significados alterados que desafían las acepciones históricas. Parafraseando a Mignolo y Appadurai, se podría decir que la semiosis de-colonial nace del reencuentro conflictivo entre la genealogía de un concepto o signo (su puesta en circulación en el mundo) y su historia (su domesticación en una práctica local).

Como observó George Marcus, la etnografía multisituada en/ del sistema-mundo implica un mayor desafío para la traducción. En la Europa del siglo xxi, confrontada con una «semiosis de-colonial», el esfuerzo de traducción consiste en relacionar o crear convergencias, homologías o equivalencias entre genealogías e historias dispares de conceptos en movimiento. Tal esfuerzo constante de negociación semiótica es una situación vivida como nueva por muchos países europeos, cuya experiencia de interculturalidad se ha situado históricamente más bien fuera del territorio nacional, en los confines de Europa como parte de su historia colonial. Si antes las tensiones semióticas eran más bien un problema de las periferias del sistema mundo, ahora se ubican en el seno del sistema y lo que antes era un problema exótico se ha convertido en un problema interno.

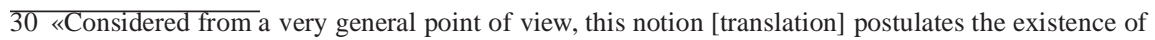
a single field of significations, concerns and interests, the expression of a shared desire to arrive at the same result... Translation involves creating convergences and homologies by relating things that were previously different» (Michel Callon, p. 32); «translation is the process or the work of making two things that are not the same, equivalent», de: John Law y John Hassard (eds.), Actor Network Theory and After (Oxford: Blackwell, 2006) 8. 